\title{
Development of autoimmunity to transglutaminase $C$ in children of patients with type 1 diabetes: relationship to islet autoantibodies and infant feeding
}

\author{
S. Hummel • M. Hummel • J. Banholzer • D. Hanak • \\ U. Mollenhauer • E. Bonifacio • A. G. Ziegler
}

Received: 27 June 2006 / Accepted: 17 October 2006 / Published online: 14 December 2006

(C) Springer-Verlag 2006

\begin{abstract}
Aims/hypothesis Coeliac disease and transglutaminase antibodies are common in patients with type 1 diabetes and their relatives. We investigated the development of transglutaminase antibodies and analysed potential risk factors for coeliac disease autoimmunity in first-degree relatives of patients with type 1 diabetes.

Methods The study was conducted by prospective observational follow-up from birth of 1,511 children at increased risk of type 1 diabetes or coeliac disease born in Germany between 1989 and 2000. Mean follow-up was to age 7.6 years. Children were tested for transglutaminase and islet autoantibodies. Children were classified as transglutaminase antibody-positive if antibodies were detected by both ELISA and radiobinding assays.

Results The risk of developing transglutaminase antibodies was $4.9 \%$ by age 8 years $(n=63 ; 95 \%$ CI, $3.7-6.1 \%)$. Transglutaminase antibodies developed at an older age than islet autoantibodies (median age, 4.9 vs 2.3 years; $p<0.005$ ), and only three children developed both transglutaminase antibodies and islet autoantibodies. Multivariate analysis indicated an increased risk of transglutaminase antibodies in children with the $H L A-D R B 1 * 03$ allele (hazard ratio for heterozygous DR3, 5.5; 95\% CI, 2.910.2; hazard ratio for homozygous DR3, 16.2; 95\% CI, $6.7-39 ; p<0.0001)$ and in children with impaired uterine
\end{abstract}

S. Hummel · M. Hummel · J. Banholzer · D. Hanak •

U. Mollenhauer A A. G. Ziegler $(\bowtie)$

Diabetes Research Institute,

Munich, Germany

e-mail: anziegler@1rz.uni-muenchen.de

E. Bonifacio

Immunology of Diabetes Unit, Istituto Scientifico San Raffaele, Milan, Italy growth (birth weight $\leq 1$ st percentile, hazard ratio, 3.1; 95\% CI, 1.1-7.8, $p=0.03$ ). Neither breast-feeding or its duration nor the age of first exposure to gluten was associated with the risk of developing transglutaminase antibodies.

Conclusions/interpretation Coeliac disease autoimmunity is initiated later than islet autoimmunity in children who are at risk. An influence of infant nutrition on the development of coeliac disease autoimmunity could not be confirmed in this prospective study.

Keywords Coeliac disease - Gluten · Islet autoantibodies . Transglutaminase $\cdot$ Type 1 diabetes

\section{Abbreviations \\ DAISY Diabetes Autoimmunity Study in the Young \\ IA-2 tyrosine phosphatase-like protein \\ TGC transglutaminase $\mathrm{C}$}

\section{Introduction}

Relatives of patients with type 1 diabetes are at increased risk of coeliac disease and coeliac disease-associated autoimmunity marked by transglutaminase C (TGC, also known as TGM2) antibodies [1-3]. Coeliac disease is induced by prolamines (gluten) present in wheat, barley or rye [4] in genetically susceptible individuals. Timing of the introduction of gluten into the infant diet, the amount of gluten consumed and the introduction of gluten after cessation of breast-feeding have been linked to the risk of coeliac disease and type 1 diabetes [5-10].

For type 1 diabetes, two prospective cohort studies (BabyDiab [8] and DAISY [Diabetes Autoimmunity in the Young] [9]) have demonstrated that exposure to gluten or 
cereals before age 3 months increases the risk of islet autoimmunity, whereas a third found no association [11]. These investigators have also looked into the possible association between the timing of gluten exposure and the risk of appearance of TGC antibodies. Preliminary analysis of data from the BabyDiab study found a trend towards an increased risk of TGC antibodies in children who received gluten early, but the conversion rate to TGC antibodies was low at the time, with 27 positive cases. In DAISY, it was recently reported that children had a fivefold increased risk of TGC antibodies when exposed to gluten-containing foods in the first 3 months of life. Now, with up to 14 years of prospective follow-up data and 63 TGC antibodypositive cases, we have re-examined the natural history of coeliac disease in relation to the development of islet autoantibodies and factors that influence the development of TGC antibodies in the BabyDiab cohort.

\section{Subjects and methods}

Subjects The BabyDiab study was commenced in 1989 to examine the natural history of islet autoimmunity and coeliac disease autoimmunity in offspring of parents with type 1 diabetes. BabyDiab prospectively follows offspring of mothers and/or fathers with type 1 diabetes from birth with venous blood sampling and collection of questionnaire data at birth and the ages of 9 months and 2, 5, 8, 11 and 14 years [12]. Antibodies to TGC and to the islet antigens insulin, glutamic acid decarboxylase and tyrosine phosphatase-like protein (IA2, also known as PTPRN) were measured in 1,511 offspring in all prospectively collected samples. HLA-DR and -DQ genotypes were determined in 1,461 offspring. A blood sample in which TGC antibodies were measured was also obtained from 1,479 mothers and 1,390 fathers. Written informed consent was obtained from the parents. The study was approved by the ethical committee of Bavaria, Germany (Bayerische Landesärztekammer no. 95357).

Autoantibody testing IgA TGC antibodies were measured by ELISA according to the manufacturer's instructions (Eurospital, Trieste, Italy). The 99th percentile of control children without diabetes and coeliac disease was used to define a positive TGC antibody result. For samples with values above this threshold, a radiobinding assay measuring IgA TGC antibodies was also performed as described previously [1]. Samples with values above the 99th centile of control children in both assays were defined as positive for TGC antibodies. Children were defined as TGC antibody-positive if they had at least one positive sample. Small bowel biopsy was offered to children with TGC antibodies and was obtained from 19 TGC antibodypositive children. Antibodies to insulin, glutamic acid decarboxylase and IA-2 were determined as described previously [12]. Islet autoantibody positivity was defined as being confirmed positive (two consecutive samples) for one or more islet autoantibodies [8].

HLA typing HLA class II alleles HLA-DRB1, HLA-DQA1 and HLA-DQB1 were determined using PCR-amplified DNA and non-radioactive sequence-specific oligonucleotide probes as described previously [1].

Questionnaire and interviews Birth weight, gestational age and sex were obtained from each child's paediatric 'passport'. Birth weight was adjusted for sex and gestational age and expressed as a percentile of the reference German population. Infant diet data were collected by questionnaire at the ages of 9 months and 2 years and by telephone interviews [8]. Families did not receive dietary advice other than that normally provided to parents in Germany. Data on the family history of coeliac disease were collected at each follow-up visit.

Statistical analysis Cumulative frequencies of TGC antibodies were determined using life table analysis with follow-up calculated from birth to the age at the first sample that was TGC antibody-positive or to the last negative sample visit [8]. The Cox proportional hazards model was used to compare outcomes for participants with

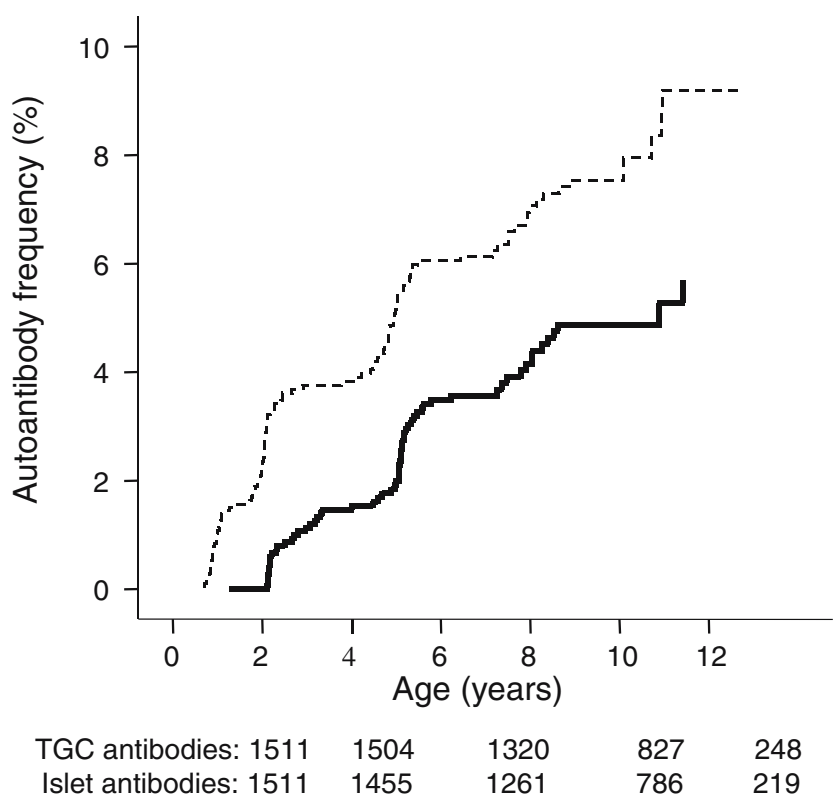

Fig. 1 Risk of development of autoantibodies in children of parents with type 1 diabetes participating in the BabyDiab study. The two curves represent different outcomes. The solid curve shows the cumulative frequency of transglutaminase antibodies in the 1,511 children and the dashed curve the cumulative frequency of islet autoantibodies in the 1,511 children. Numbers below the abscissa indicate the number of antibody-negative children remaining on follow-up with regard to age 
different covariate/factor categories. Variables analysed included: HLA-DR3 status (DR3/3; DR3/ $x$, where $x$ is an allele other than DR3; no DR3 allele); birth weight centile ( $\leq 1$ st percentile; 2 nd to 10 th percentile; $>10$ th percentile); parental coeliac status (coeliac disease; TGC antibodies); total duration of breast-feeding (none; 1 week to 3 months; 3.1-6 months; >6 months); food supplements received before age 3 months (none [breast-milk only]; supplementation with formula milk-based foods only; supplementation with gluten-free solid foods; supplementation with glutencontaining foods); age at first gluten exposure, defined as intake of foods containing wheat, rye, barley or oats (before age 3 months; between 3 and 6 months; after 6 months). Hazard ratios are from univariate analysis unless otherwise stated. All $p$ values are two-tailed. Statistical analyses were performed using SPSS (SPSS, Chicago, IL, USA).

\section{Results}

Sixty-three offspring developed TGC antibodies, including three who also developed islet autoantibodies (cumulative risk of developing TGC antibodies by age 8 years, $4.9 \%$; 95\% CI, 3.7-6.1\%; Fig. 1). Another 104 cases developed islet autoantibodies without TGC antibodies (cumulative islet autoantibody risk by age 8 years, $7.8 \%$; 95\% CI, 6.29.4). No children had TGC antibodies before age 2 years. TGC antibodies were transient (negative in the most recent sample) in 12 children. Overall, TGC antibodies developed later than islet autoantibodies (median age 4.9 years vs 2.3; $p<0.005)$. In three children who developed both islet and TGC antibodies, islet autoantibodies developed 2.2, 2.3 and 3.2 years before TGC antibodies. Coeliac disease was confirmed by histology of a small bowel biopsy in 16 of 19 TGC antibody-positive children.

The risk of developing TGC antibodies was associated with HLA-DR3 and with very low ( $<1$ st percentile) birth weight (Table 1 ). HLA-DR3/ $x$ children had a significantly higher risk of developing TGC antibodies than DR3negative children (hazard ratio 5.7; $p<0.0001$ ). Children homozygous for DR3 had the greatest risk (hazard ratio $16.2 ; p<0.0001)$. Breast-feeding did not influence the development of TGC antibodies. Introduction of formula milk, gluten-containing or gluten-free solid food supple-
Table 1 Factors influencing transglutaminase antibody development in offspring of parents with type 1 diabetes
${ }^{a}$ Reference category for hazard ratio

\begin{tabular}{|c|c|c|c|c|c|}
\hline & $\begin{array}{l}\text { Number of } \\
\text { subjects }\end{array}$ & $\begin{array}{l}\text { Number positive } \\
\text { for TGC } \\
\text { antibodies }\end{array}$ & $\begin{array}{l}\text { Hazard } \\
\text { ratio }\end{array}$ & $95 \% \mathrm{CI}$ & $p$ value \\
\hline \multicolumn{6}{|l|}{$\overline{\text { HLA }}$} \\
\hline Non-DR3 & 904 & 13 & $1^{\mathrm{a}}$ & & \\
\hline $\mathrm{DR} 3 / 3$ & 36 & 8 & 16.2 & $6.7-39.0$ & $<0.0001$ \\
\hline $\mathrm{DR} 3 / x$ & 492 & 39 & 5.7 & $3.0-10.6$ & $<0.0001$ \\
\hline \multicolumn{6}{|l|}{ Birth weight } \\
\hline$\leq 1$ st percentile & 30 & 4 & 3.4 & $1.2-9.4$ & 0.02 \\
\hline 2nd to 10 th percentiles & 100 & 3 & 0.8 & $0.2-2.5$ & 0.7 \\
\hline$>10$ th percentile & 1,200 & 47 & $1^{\mathrm{a}}$ & & \\
\hline \multicolumn{6}{|l|}{ Parental coeliac disease } \\
\hline Parent with coeliac disease & 12 & 1 & 2.4 & $0.3-17.3$ & 0.4 \\
\hline $\begin{array}{l}\text { Neither with coeliac } \\
\text { disease }\end{array}$ & 1,493 & 62 & $1^{\mathrm{a}}$ & & \\
\hline \multicolumn{6}{|c|}{ Total breast-feeding time (months) } \\
\hline 0 & 272 & 7 & 0.6 & $0.2-1.5$ & 0.3 \\
\hline $0.1-3$ & 359 & 20 & 1.3 & $0.6-2.7$ & 0.5 \\
\hline $3.1-6$ & 253 & 11 & $1^{\mathrm{a}}$ & & \\
\hline$>6$ & 466 & 16 & 0.8 & $0.4-1.8$ & 0.6 \\
\hline \multicolumn{6}{|c|}{ Food supplements first 3 months } \\
\hline No supplements & 653 & 24 & $1^{\mathrm{a}}$ & & \\
\hline Formula milk-based only & 503 & 23 & 1.2 & $0.7-2.1$ & 0.6 \\
\hline Non-gluten solid foods & 45 & 3 & 1.8 & $0.5-6.0$ & 0.3 \\
\hline Gluten & 18 & 1 & 1.5 & $0.2-10.9$ & 0.7 \\
\hline \multicolumn{6}{|c|}{ Age at gluten introduction (months) } \\
\hline$<3$ & 18 & 1 & 1.6 & $0.2-12.1$ & 0.7 \\
\hline $3.1-6$ & 384 & 13 & $1^{\mathrm{a}}$ & & \\
\hline$>6$ & 754 & 38 & 1.4 & $0.8-2.7$ & 0.2 \\
\hline \multicolumn{6}{|l|}{ Sex of the child } \\
\hline Male & 773 & 26 & $1^{\mathrm{a}}$ & & \\
\hline Female & 738 & 37 & 1.5 & $0.9-2.5$ & 0.1 \\
\hline
\end{tabular}


ments during the first 3 months of life was not associated with an increased risk of TGC antibodies. Delaying the introduction of gluten-containing foods until after age 6 months did not increase the risk of developing TGC antibodies. Whether children were still breast-fed at the first introduction of gluten did not affect the risk of developing TGC antibodies (data not shown). Parental coeliac disease, reported in three parents and found as TGC antibody positivity in a further nine parents, was not significantly associated with an increased risk of developing TGC antibodies in the child.

By multivariate analysis, HLA-DR3/x (adjusted hazard ratio, 5.5; 95\% CI, 2.9-10.2; $p<0.0001$ ), HLA-DR3/DR3 (adjusted hazard ratio, $16.2 ; 95 \% \mathrm{CI}, 6.7-39 ; p<0.0001$ ) and birth weight below the first percentile (adjusted hazard ratio, $3.1 ; 95 \%$ CI, 1.1-7.8; $p=0.03$ ) were associated with increased risk of developing TGC antibodies (adjusted for sex, maternal type 1 diabetes and parental coeliac disease status). When outcome was restricted to children who had confirmed and persistent TGC antibodies (persistent positivity for TGC antibodies up to the last sample available), HLA-DR $3 / x$ (adjusted hazard ratio, 5.4; 95\% CI, 2.4-12.1; $p<0.0001$ ), HLA-DR3/DR3 (adjusted hazard ratio, 22.8; $95 \%$ CI, 8.2-63; $p<0.0001$ ) and birth weight below the first percentile (adjusted hazard ratio, 5.9; 95\% CI, 2.0-17.3; $p=0.001)$ were associated with increased TGC antibody risk.

\section{Discussion}

This prospective study shows a high risk of developing TGC antibodies in offspring of parents with type 1 diabetes, particularly in children with the DR3-DQ2 haplotype. An increased risk of coeliac disease in patients with type 1 diabetes and their relatives compared with the general population is well documented [1-3]. It has been suggested that untreated coeliac disease enteropathy is a risk for type 1 diabetes and that type 1 diabetes could be prevented in individuals with coeliac disease if coeliac disease were diagnosed and treated early [11]. Prospective data indicate that islet autoimmunity occurs early in at-risk individuals and prior to TGC antibodies and coeliac disease. Thus, it appears unlikely that treating coeliac disease will prevent the development of islet autoantibodies in children who are genetically at risk. Furthermore, pilot studies in islet autoantibody-positive individuals have failed to provide evidence that type 1 diabetes can be prevented by a glutenfree diet [13].

We have previously reported an increased risk of developing islet autoantibodies in genetically at-risk children who receive gluten before age 3 months [8]. We still observe this relationship with longer follow-up, and now find an increased risk of developing type 1 diabetes in these children (data not shown). The relationship between early gluten exposure and islet autoantibodies is consistent between our study and the DAISY cohort [9]. We were unable, however, to confirm the relationship between the timing of gluten exposure and TGC antibody risk recently reported in the DAISY cohort. Cohort size and case numbers were similar between the studies. Infant feeding practices differ considerably between the USA and Germany and could account for the inconsistency. It is also possible that the inconsistency contributes to the differences in data collection methods and follow-up. In contrast to retrospective case-control studies, both prospective studies consistently find no protective effect of breast-feeding on the development of TGC antibodies and coeliac disease.

In summary, the BabyDiab study does not confirm an association of early infant diet with the initiation of coeliac disease-associated autoimmunity.

Acknowledgements We thank Andrea Baumgarten for expert technical assistance and the families and paediatricians in Germany who participated in the study. We thank E. Bravi (Eurospital, Trieste, Italy) for advice regarding the IgA-TGC measurements. This study was supported by grants from the German Society for Coeliac Disease (Deutsche Zöliakiegesellschaft), Juvenile Diabetes Research Foundation (JDRF no. 1-2003-646 and no. 1-2006-665), the Foundation 'Children with type 1 diabetes' (Stiftung 'Das Zuckerkranke Kind') and the Deutsche Diabetesgesellschaft.

Duality of interest The authors are not aware of any duality of interest.

\section{References}

1. Hummel M, Bonifacio E, Stern M, Dittler J, Schimmel A, Ziegler AG (2000) Development of celiac disease-associated antibodies in offspring of parents with type 1 diabetes. Diabetologia 43:1005-1011

2. Williams AJ, Norcross AJ, Lock RJ, Unsworth DJ, Gale EA, Bingley PJ (2001) The high prevalence of autoantibodies to tissue transglutaminase in first-degree relatives of patients with type 1 diabetes is not associated with islet autoimmunity. Diabetes Care 24:504-509

3. Saukkonen T, Ilonen J, Akerblom HK, Savilahti E (2001) Prevalence of coeliac disease in siblings of patients with type I diabetes is related to the prevalence of $D Q B 1^{*} 02$ allele. Diabetologia 44:1051-1053

4. Trier JS (1991) Celiac sprue. N Engl J Med 325:1709-1719

5. Norris JM, Barriga K, Hoffenberg EJ et al (2005) Risk of celiac disease autoimmunity and timing of gluten introduction in the diet of infants at increased risk of disease. JAMA 293:23432351

6. Weile B, Cavell B, Nivenius K, Krasilnikoff PA (1995) Striking differences in the incidence of childhood celiac disease between Denmark and Sweden: a plausible explanation. J Pediatr Gastroenterol Nutr 21:64-68

7. Ivarsson A, Hernell O, Stenlund H, Persson LA (2002) Breastfeeding protects against celiac disease. Am J Clin Nutr 75:914921

8. Ziegler AG, Schmid S, Huber D, Hummel M, Bonifacio E (2003) Early infant feeding and risk of developing type 1 diabetesassociated autoantibodies. JAMA 290:1721-1728 
9. Norris JM, Barriga K, Klingensmith G et al (2003) Timing of cereal exposure in infancy affects risk of islet autoimmunity. The Diabetes Autoimmunity Study in the Young. JAMA 290:1713-1720

10. Ventura A, Magazzu G, Greco L (1999) Duration of exposure to gluten and risk for autoimmune disorders in patients with celiac disease. Gastroenterology 117:297-303

11. Virtanen SM, Kenward MG, Erkkola M et al (2006) Age at introduction of new foods and advanced beta cell autoimmunity in young children with HLA-conferred susceptibility to type 1 diabetes. Diabetologia 49:1512-1521
12. Ziegler AG, Hummel M, Schenker M, Bonifacio E (1999) Autoantibody appearance and risk for development of childhood diabetes in offspring of parents with type 1 diabetes: the 2-year analysis of the German BABYDIAB Study. Diabetes 48:460481

13. Pastore MR, Bazzigaluppi E, Belloni C, Arcovio C, Bonifacio E, Bosi E (2003) Six months of gluten-free diet do not influence autoantibody titers, but improve insulin secretion in subjects at high risk for type 1 diabetes. J Clin Endocrinol Metab 88:162165 